\title{
LIGHT-INDUCED MODIFICATION OF 3D PHOTONIC BAND STRUCTURE DETECTED BY MEANS OF PHOTOREFLECTION
}

\author{
A.M. Kapitonov, S.M. Kachan, A.N. Ponyavina, S.V. Gaponenko \\ Institute of Molecular and Atomic Physics, National Academy of Science of Belarus \\ F. Skaryna Ave. 70, Minsk 220072, Belarus
}

V.N. Bogomolov, A.V. Prokofiev

Ioffe Physico-Technical Institute, Russian Academy of Science

Politekhnicheskaya St. 26, St.-Petersburg 194021, Russia

P. SitAReK AND J. MisieWiCZ

Institute of Physics, Wrocław Technical University

Wybrzeże Wyspiańskiego 27, 50-370 Wrocław, Poland

(Received September 8, 1998; in final form January 11, 1999)

Porous synthetic opal possessing a three-dimensional photonic band structure of semimetallic type was impregnated with polycrystalline CdS. The photonic stop band in (111) direction was examined by means of photoreflection technique. Under $\mathrm{cw}$ laser excitation of semiconductor inclusions the reflectance of the system changes indicating a modification of photonic band structure. A possible mechanism is discussed. Numerical simulations within the framework of quasicrystalline approximation are given.

PACS numbers: $42.70 . \mathrm{Qs}, 42.70 . \mathrm{Gi}$

\section{Introduction}

Wave nature of light allows us to control optical properties of materials by means of purposeful using of scattering and interference of waves. Alone with incoherent scattering, Bragg-like reflections can appear, when components of material are spatially ordered. Dielectric media with periodic index of refraction are usually referred to as photonic crystals due to the analogy with periodic potential inherent in crystalline solids. Starting from pioneering works by Yablonovitch and John [1,2] several intriguing theoretical predictions [3] were made, e.g., on 
the possibility to modify spontaneous emission of light or to build a thresholdless laser. The visible and near infra-red (IR) range is commonly expected to be a preferable spectral region for future applications of photonic crystals. But the problem of practical realization of a three-dimensional superlattice of different dielectrics with a period of hundreds of nanometers arises. To our knowledge, at present submicron lithography techniques enable us to fabricate only a few layers of periodic structures [4] appropriate for near IR, and laser-induced direct-write deposition from the gas phase can be suitable for fabrication of far IR (periods of about $100 \mu \mathrm{m}$ ) 3D photonic structures [5].

A promising approach is to use the growth and self-assembling of monodisperse colloidal particles. This process can result in formation of colloidal crystals [6]. Synthetic opal seems to be one of the most advanced materials fabricated by means of such technique. It consists of uniform silica submicron globules close-packed usually with the symmetry of the face centered cubic type (f.c.c.). After appropriate treatment $[7,8]$ this ordered ensemble of globules acquires a considerable mechanical durability. In this way macroscopic photonic crystals were made with following important properties: (i) they are entirely solid-state; (ii) they possess three-dimensional periodicity of the index of refraction; (iii) a characteristic size of structure unit (silica globule) is in the range of a few hundred nanometers that is relevant to the visible light wavelength.

Well-known iridescent colors of opal are nothing else but Bragg-like diffraction, when photons with a certain 4-vector of energy and momentum $(\hbar k, i \hbar \omega / c)$ are unable to propagate inside a periodic dielectric medium. Such photonic stop bands occur at spectral and spatial positions which depend on the symmetry of crystal, on the values of refraction indices, and on the shape and volume fractions of all different components of the crystal unit cell. Under certain conditions [9], stop bands can overlap over all possible directions at certain frequency and omnidirectional photonic band gap will open up. This will be the case analogous to the electronic band gap in semiconductors and insulators, when electronic waves of certain energy cannot propagate in the medium with periodic potential. But optical contrast of opals $\left(n_{\text {air }}=1.0\right.$ for air between particles and $1.25 \leq n_{\text {glob }} \leq 1.45$ for silica globules, whose index of refraction varies because of their porosity) allows semimetallic type of photonic band structure only, when photonic stop bands corresponding to different crystallographic directions have different spectral positions and widths, which is shown in more detail elsewhere $[10,11]$.

A way to improve the situation is to fill with highly refractive matter the cavities between silica globules of opal, which are originally empty. Of course, it is more interesting to deal with entirely solid state structures without any fluid components. This can be made by means of sol-gel technique, which provides filling of pores with various oxides, e.g., $\mathrm{TiO}_{2}$ [12]. The study of various types of nonlinearities in photonic crystals is rather important and it is a topical problem [13-15]. Particularly, it can lead to development of new types of switches [16], media with special conditions for soliton propagation [17], etc. The aim of the present work was to produce a light sensitive photonic crystal on the base of artificial opal and to detect a response of such a superlattice to optical excitation. 


\section{Sample preparation and characterization}

Semiconductors look to be convenient fillers of opal voids because their refraction index can exceed even 3 in the visible range and their properties are rather sensitive to illumination. CdS was chosen in the way to satisfy the requirement that photonic stop bands of superlattice must be inside the spectral region of transparency of semiconductor inclusions (edge of fundamental absorption of bulk CdS is nearby $500 \mathrm{~nm}$ and index of refraction is 2.5). Vapor phase synthesis with subsequent annealing in $\mathrm{Cd}$ or $\mathrm{S}$ atmosphere was used to deposit $\mathrm{CdS}$ into the pores of initial opal matrix. As a result of this procedure was the formation of polycrystalline semiconductor between silica globules. Figure 1 presents transmission

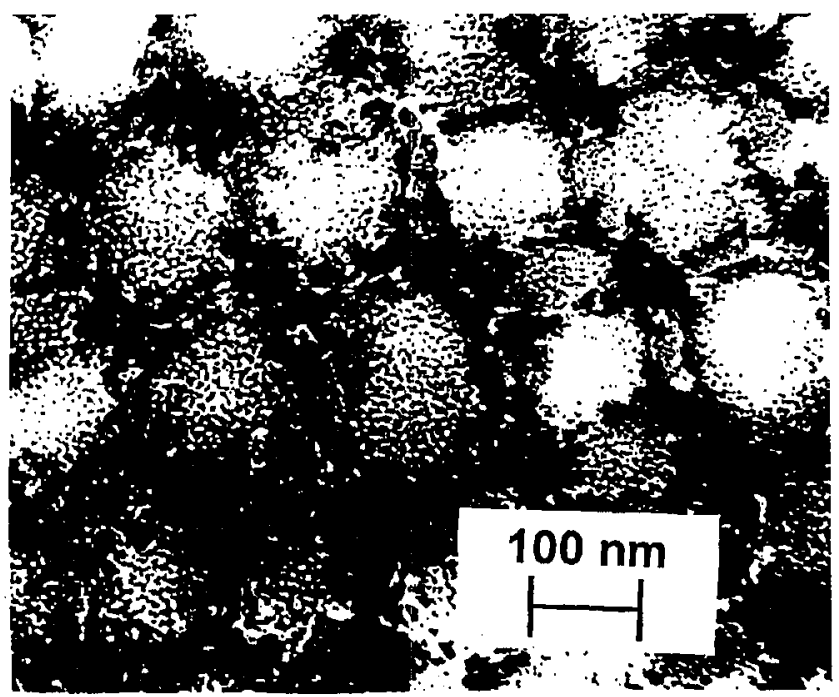

Fig. 1. TEM-image of opal matrix (fair globules) filled with CdS. Black regions depict to semiconductor inclusions.

electron microscope image of opal with CdS. The arranged silica globules, which appear as light spots, exhibit their intrinsic porous substructure. Dark aggregations around them correspond to semiconductor inclusions. Additional studies [18] have shown that a size of CdS crystallites (average value $\sim 10 \mathrm{~nm}$ ) is bigger than the exciton Bohr radius in this material $\left(a_{\mathrm{B}}=2.8 \mathrm{~nm}\right)$. Consequently, the semiconductor phase occurs in the so-called regime of weak quantum confinement [19]. Significant changes of optical characteristics under optical excitation or application of electric field [19] are inherent in this regime.

\section{Optical technique}

A standard arrangement of photoreflectance apparatus was used. This technique is widely used in optical characterization of semiconductor structures [20], enabling the investigation of built-in electric fields, doping concentrations, critical point energies, and so on. A $488 \mathrm{~nm}$ line of argon laser was applied as a pump beam source, the power was $5 \mathrm{~mW} / \mathrm{cm}^{2}$, the frequency of modulation was $120 \mathrm{~Hz}$. 
As a probe beam we used a light passed through'a double grating monochromator. In front of the probe beam detector (photodiode) a glass filter was mounted to eliminate the scattered laser light. A detailed description of set-up can be found in Ref. [21].

\section{Results}

As was mentioned above, photonic pseudogap inherent in opal-based photonic crystals can be modified by means of filling of opal pores with various materials. Changing of average refraction index of interglobule space causes a spectral shift of photonic stop band. This is demonstrated in Fig. 2, where the evolution of the transmission spectrum measured in (111) direction of the same opal plate, but filled with various liquids, is presented. Additionally, photonic stop band becomes more pronounced when an optical contrast (ratio of refraction indices of silica globules and of interglobule spacing) increases.

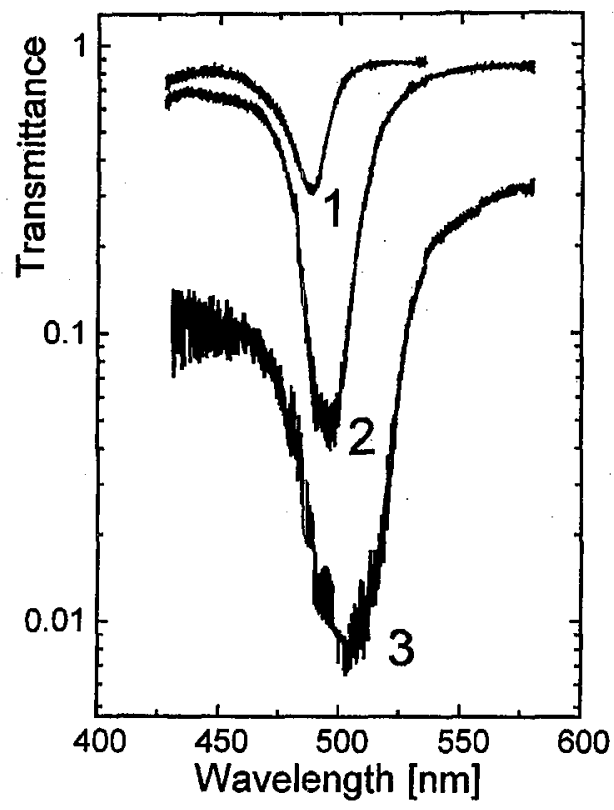

Fig. 2. Transmission of the same opal plate impregnated with liquids possessing different refraction indices: 1 - ethanol $(n=1.361), 2$ - cyclohexane $(n=1.426)$, 3 - toluene $(n=1.497)$.

Photonic pseudogap of CdS-in-opal superlattice and light-induced modification of the latter are depicted in Fig. 3. The lower panel of Fig. 3 displays a peak of reflectivity of opal" filled with CdS. It conforms photonic stop band in (111) crystallographic direction of this superlattice. The upper panel of Fig. 3 shows a normalized variation of reflectivity, $\Delta R / R$, when the superlattice is illuminated by $\mathrm{Ar}^{+}$laser. $R$ is the initial (dark) reflectivity and $\Delta R$ is its change under laser excitation. 


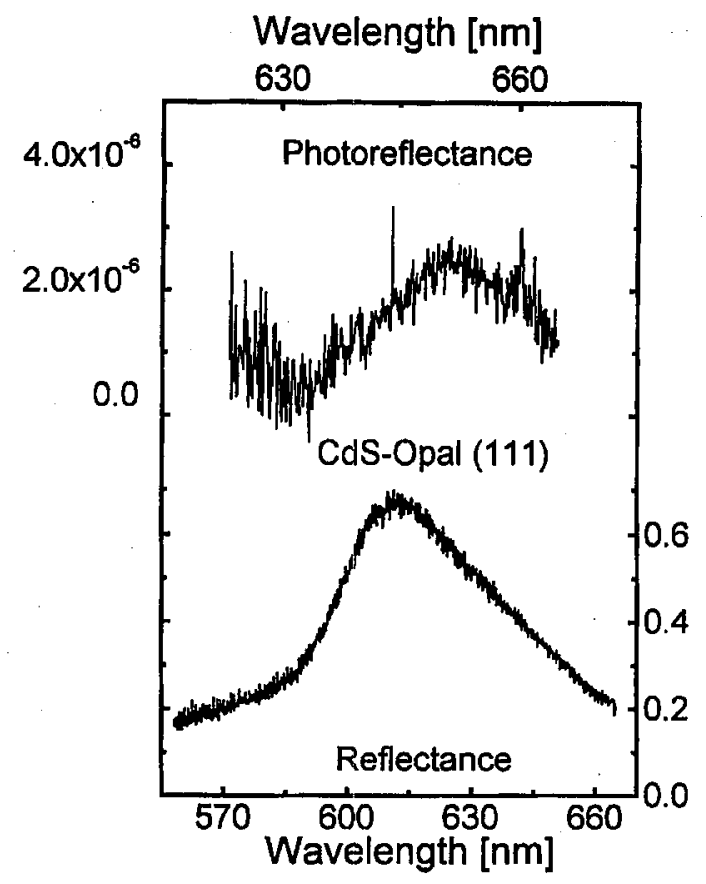

Fig. 3. The lower panel: linear reflectance of photonic superlattice consisting of opal matrix with CdS inclusions. The upper panel: photoreflectance of this structure under laser excitation.

The shape of $\Delta R / R$ curve suggests a red shift of photonic stop band. Such a reply of superlattice is indicative to the growth of refraction index of semiconductor inclusions under laser excitation (compare with Fig. 2). This growth can have a thermal origin. Really, energy of a single photon $\left(4.07 \times 10^{-19} \mathrm{~J}\right)$, being scattered inside CdS crystallite of size $5 \div 10 \mathrm{~nm}$, must rise the temperature roughly by $1 \mathrm{~K}$. This is enough for an increase in index of refraction of semiconductor by $\Delta n \sim 10^{-4}$ [22]. Numerical simulations (see below) show that such value of $\Delta n$ is sufficient to cause a modification of photonic stop band similar to the one shown in Fig. 3.

\section{Numerical simulation}

A quite adequate approach to describe spectral features of transmission and reflection of opal-based superlattices is a model of multilayer scattering medium consisting of correlated scatterers $[10,11]$. It is based on statistical multiple wave scattering theory [23] and enables us to evaluate coherent transmission and reflection spectra. Cooperative effects in a single monolayer, namely, coherent rescattering on particles and interference of the scattered waves are treated within the quasicrystalline approximation [24]. Then coherent fields of single layers are given by the scattering amplitudes $\boldsymbol{F}^{ \pm}$

$$
\boldsymbol{F}^{ \pm}=-e \frac{\pi}{k^{2}} \sum_{l} \rho( \pm 1)^{l}(2 l+1)\left(c_{l} \pm d_{l}\right)
$$


where $k$ is a wave-vector of the incident plane monochromatic wave, the plus and minus signs correspond to the transmitted and reflected waves, respectively, and $\rho$ is a surface concentration of particles. The coefficients $c_{l}$ and $d_{l}$ are based on Mie theory, but coherent irradiation of particles within a monolayer is accounted for $[24,11]$.

In a multilayer system, not only the incident radiation field and the fields scattered by other particles of the same monolayer contribute to the effective field for a given particle, but the fields from particles of other layers are also contributed to this effective field. Assuming individual monolayers to be statistical independent, the coherent fields can be written as follows:

$$
\begin{aligned}
& \left\langle\boldsymbol{E}^{+}(\boldsymbol{z})\right\rangle=\exp (\mathrm{i} k|z|)\left[e+\sum_{j=1}^{N} \boldsymbol{G}_{j}^{+}\right], \\
& \left\langle\boldsymbol{E}^{-}(z)\right\rangle=\exp (\mathrm{i} k|z|) \sum_{j=1}^{N} \boldsymbol{G}_{j}^{-} \exp \left[(j-1) 2 \mathrm{i} k l_{m}\right],
\end{aligned}
$$

where $e$ is a unit polarization vector of the incident wave, $G_{j}^{ \pm} \equiv G_{j}\left( \pm z_{0}\right)$ are the forward and backward scattering amplitudes of the $j$-th monolayer in the presence of other monolayers, $l_{m}$ is the interlayer spacing, and $z$ is the direction of propagation. In order to evaluate the coherent transmission coefficient $T^{k}=$ $\left|\left\langle\boldsymbol{E}^{+}(z)\right\rangle\right|^{2}$ and the coherent reflection coefficient $R^{k}=\left|\left\langle\boldsymbol{E}^{-}(z)\right\rangle\right|^{2}$ one should solve a system of equations for self-consistent field

$$
\begin{aligned}
& \boldsymbol{G}_{m}^{+}=\boldsymbol{F}^{+}+\boldsymbol{F}^{+} \sum_{j-1}^{m-1} \boldsymbol{G}_{j}^{+}+\boldsymbol{F}^{-} \sum_{j=m+1}^{N} \boldsymbol{G}_{j}^{-} \exp \left[(j-m) 2 \mathrm{i} k l_{m}\right], \\
& \boldsymbol{G}_{m}^{-}=\boldsymbol{F}^{-}+\boldsymbol{F}^{-} \sum_{j-1}^{m-1} \boldsymbol{G}_{j}^{+}+\boldsymbol{F}^{+} \sum_{j=m+1}^{N} \boldsymbol{G}_{j}^{-} \exp \left[(j-m) 2 \mathrm{i} k l_{m}\right],
\end{aligned}
$$

where $m$ is the number of layer. When $G_{m}^{ \pm}$are found they are to be substituted into Eqs. $(2,3)$.

Numerical calculations have been carried out for a layered system consisting of close-packed monolayers of spherical particles. The refractive index of the spheres was taken to be equal to $n_{\text {sphere }}=1.26$, which is close to the effective refraction index of silica clusters in the artificial opals. The refraction index of interglobule spacing was chosen to be $n_{\text {medium }}=2.00$, it looks quite reasonable for the case of partial filling with semiconductor. The density of particle arrangement within a given monolayer was close to a maximum (the overlapping factor $\eta=\rho \pi d^{2} / 4=0.55$ ). The interlayer spacing defined as a distance between two planes connecting centers of particles in two neighboring monolayers was taken to be equal to the particle diameter $d$. Coherent reflection of the system is presented at the lower panel of Fig. 4 for a set of 100 monolayers. The shape and spectral position of the peak correlates with that from experiment (see Fig. 3, the lower part). And what happens when the refractive index of interglobule medium changes slightly is shown on the upper panel of Fig. 4 . The variation of refractive 


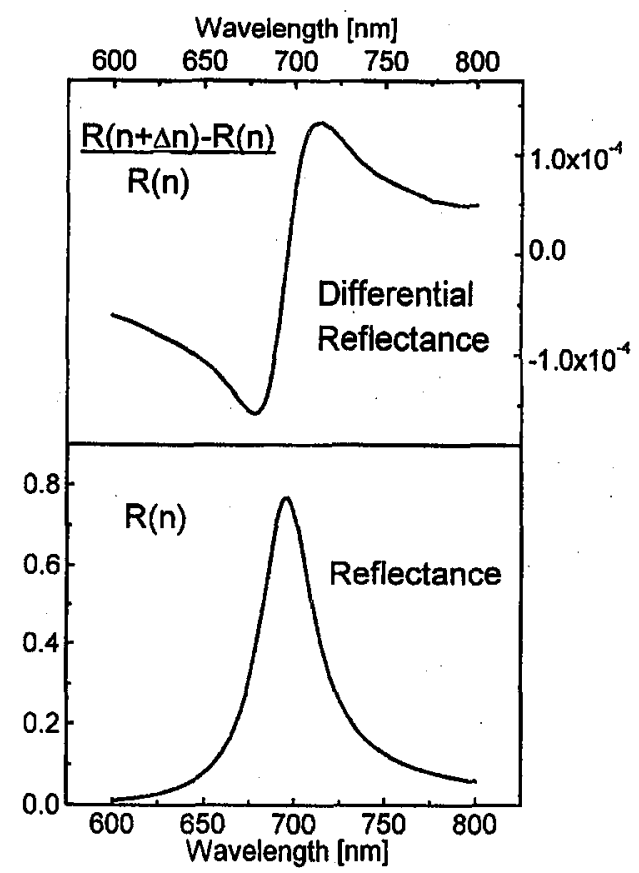

Fig. 4. Numerical modeling of reflectivity of opal-based light-sensitive photonic crystal. The lower panel shows a linear reflection. The upper panel gives a normalized change of reflectivity $[R(n+\Delta n)-R(n)] / R(n)$ in the case of an increase in refraction index of interparticle medium $\Delta n=10^{-5}$.

index was taken $\Delta n_{\text {medium }}=10^{-5}$. Then the spectra of coherent reflection $R(n)$ and $R(n+\Delta n)$ were calculated. The value of $[R(n+\Delta n)-R(n)] / R(n)$ is plotted in the figure which is an analog of experimentally measured curve from Fig. 3 (the upper part). The same red shift of photonic stop band is clearly distinguishable.

\section{Conclusion}

In conclusion, silica-based 3D superlattice with light-sensitive semiconductor inclusions was synthesized and characterized. It exhibits the photonic band structure in the visible range. Numerical modeling of the light propagation through such a system within the frame of quasicrystalline approximation is in good agreement with experimental data. Under laser illumination reflectivity of the superlattice changes indicating light-induced modification of photonic pseudogap. Numerical simulations confirm the data obtained by means of photoreflectance technique. Such behavior of photonic stop band suggests an increase in refractive index of interglobule spacings, which are filled with CdS. A possible mechanism of this increase has a thermal source, namely, heating of semiconductor by absorbed laser light. At the next stage of our studies, transient photo-induced tuning of a photonic crystal by means of optical nonlinearities will be investigated. 


\section{References}

[1] E. Yablonovitch, Phys. Rev. Lett. 58, 2059 (1987).

[2] S. John, Phys. Rev. Lett. 58, 2486 (1987).

[3] See, e.g., Photonic Band Gap Materials, special issue of J. Opt. Soc. Am. B 10, (2) (1993); Photonic Band Structures, special issue of J. Mod. Opt. 41, (2) (1994); J.D. Janopoulos, R.D. Meade, J.N. Winn, Photonic Crystals: Molding the Flow of Light, Princeton Univ. Press, Princeton 1995.

[4] A. Scherer, C.C. Cheng, O. Painter, J. O'Brien, A. Yariv, E. Yablonovitch, V. Arbet-Engles, in: Proc. XXIII Int. Conf. Phys. Semicond., Berlin 1996, Eds. M. Scheffler, R. Zimmermann, World Scientific, Singapore 1996, p. 3063.

[5] M.C. Wanke, O. Lehmann, K. Müller, Q. Wen, M. Stuke, Science 275, 1284 (1997).

[6] P. Pieranski, Contemp. Phys. 24, 25 (1983).

[7] V.N. Bogomolov, D.A. Kurdyukov, A.V. Prokofiev, S.M. Samoilovich, Pis'ma Zh. Exp. Teor. Fiz. 63, 496 (1996) [JETP Lett. 63, 520 (1996)].

[8] A.L. Rogach, A. Kornowski, O.E. Rogach, A.M. Kapitonov, N.V. Gaponenko, S.V. Gaponenko, A. Eychmüller, in preparation.

[9] R. Biswas, M.M. Sigalas, G. Subramania, K.-M. Ho, Phys. Rev. B 57, 3701 (1998).

[10] V.N. Bogomolov, S.V. Gaponenko, A.M. Kapitonov, A.V. Prokofiev, A.N. Ponyavina, N.I. Silvanovich, S.M. Samoilovich, Appl. Phys. A 6, 613 (1996).

[11] V.N. Bogomolov, S.V. Gaponenko, I.N. Germanenko, A.M. Kapitonov, E.P. Petrov, N.V. Gaponenko, A.V. Prokofiev, A.N. Ponyavina, N.I. Silvanovich, S.M. Samoilovich, Phys. Rev. E 55, 7619 (1997).

[12] A.M. Kapitonov, N.V. Gaponenko, V.N. Bogomolov, A.V. Prokofiev, S.M. Samoilovich, S.V. Gaponenko, Phys. Status Solidi A 165, 119 (1998).

[13] S. John, T. Quang, Phys. Rev. Lett. 76, 2484 (1996).

[14] E. Lidorikis, Q. Li, C.M. Soukoulis, Phys. Rev. E 55, 3613 (1997).

[15] J. Martorell, R. Vilaseca, R. Corbalán, Appl. Phys. Lett. 70, 702 (1997).

[16] P. Tran, Opt. Lett. 21, 1138 (1996).

[17] H. He, P.D. Drummond, Phys. Rev. Lett. 78, 4311 (1997).

[18] V.N. Astratov, V.N. Bogomolov, A.A. Kaplyanskii, A.V. Prokofiev, L.A. Samoilovich, S.M. Samoilovich, Yu.A. Vlasov, Nuovo Cimento D 17, 1349 (1995).

[19] S.V. Gaponenko, Optical Properties of Semiconductor Nanocrystals, Cambridge Univ. Press, Cambridge 1998.

[20] F.H. Pollak, O.J. Glembocki, Proc. SPIE 946, 2 (1998); N. Bottka, D.K. Gaskill, R.S. Sillmon, R. Henry, R. Grosser, J. Electron. Mater. 17, 161 (1988).

[21] J. Misiewicz, K. Jezierski, P. Sitarek, P. Markiewicz, R. Korbutowicz, M. Panek, B. Sciana, M. Tłaczała, Adv. Mater. Opt. Electron. 5, 321 (1995).

[22] Landolt-Börnstain Numerical Data and Functional Relationships in Science and Technology, New Series, Eds. O. Madelung, M. Schulz, H. Weiss, Vol. 17, Springer-Verlag, Berlin 1982.

[23] A. Ishimary, Wave Propagation and Scattering in Random Media, Academic Press, New York 1978.

[24] K.M. Hong, J. Opt. Soc. Amer. 70, 821 (1980). 